Received: 2016.11.17

Accepted: 2017.01 .08

Published: 2017.10 .20

\title{
Imaging Diagnosis of Chronic Encapsulated Intracerebral Hematoma, a Comparison of Computed Tomography (CT) and Magnetic Resonance Imaging (MRI) characteristics
}

Authors' Contribution:
A Study Design
B Data Collection
C Statistical Analysis
D Data Interpretation
E Manuscript Preparation
F Literature Search
G Funds Collection

\author{
Sainan Cai ${ }^{\text {EF }}$, Bing Zhou ${ }^{\text {BG }}$, Haiyan Liao ${ }^{\text {DF }}$, Changlian $\operatorname{Tan}^{\text {AE }}$ \\ Department of Radiology, Second Xiangya Hospital, Central South University, Changsha, Hunan, P.R. China
}

Author's address: Changlian Tan, Department of Radiology, Second Xiangya Hospital, Central South University, 139 Renmin Road, Changsha 410011, Hunan, P.R. China, e-mail: tanchanglian@yahoo.com

Source of support: This study was supported by Chinese National Nature and Science Foundation (81471646), Hunan Provence Nature and Science Foundation (14JJ2027)

\section{Summary}

Background: Chronic encapsulated intracerebral hematoma (CEICH) is a rare type of intracerebral hematoma that is often misdiagnosed.

To explore the characteristics of CEICH on computerized tomography (CT) and magnetic resonance imaging (MRI).

Material/Methods: $\quad$ Clinical, CT, MRI, and susceptibility weighted imaging (SWI) data of 5 patients who were diagnosed with CEICH on surgery and pathology were retrospectively analyzed.

Results: $\quad$ CT showed quasi-circular or elliptical lesions with clear borders in all 5 cases and iso-density or low-density in the center of lesions that were surrounded by peripheral edema in 2 cases. CT showed mass effect in 5 patients. On contrast-enhanced CT, 2 cases exhibited mild ring enhancement, and 3 cases exhibited moderate ring enhancement. MRI showed cystic lesions with high uniform signal on Tl-weighted images (T1WI) and T2-weighted images (T2WI), a lowsignal ring sign on the coated cystic lesions on T2WI, a lower signal ring sign on SWI, and ring enhancement after administration of contrast.

Conclusions: $\quad$ CT imaging of CEICH did not reveal any typical characteristics in the studied patients. MRI showed an envelope with a "ring" and intra-capsular bleeding features. MRI is an effective imaging modality for the diagnosis of CEICH.

MeSH Keywords: $\quad$ Diffusion Magnetic Resonance Imaging • Neuroimaging • Radiology

PDF file: $\quad$ http://www.polradiol.com/abstract/index/idArt/902417

\section{Background}

Chronic encapsulated intracerebral hematoma (CEICH) is a rare type of intra-cerebral hematoma. The main clinical symptoms of CEICH include a chronic, progressive development and progressive intracranial hypertension in most cases [1]. Brain imaging shows lesions of chronic scalability because of a slow clinical course and atypical clinical and imaging findings. CEICH is often misdiagnosed as glioma, metastasis, or brain abscess [2]. We retrospectively analyzed clinical and radiological data of 5 patients with CEICH that was confirmed by surgical pathology, and discuss the imaging characteristics of CEICH in order to improve the diagnosis of this disease. 


\section{Material and Methods}

\section{Patients}

Five patients were diagnosed with CEICH from 2009 to 2013 in the Department of Neurosurgery, Second Xiangya Hospital, Central South University. This study was approved by the ethics committee of Second Xiangya Hospital, Central South University. Signed informed consent was obtained from all patients. The study included 3 males and 2 females with a mean age of 40.6 years (range: $28-55$ years). The mean disease duration was 16 days, with a range of 6-30 days.

Headache or sudden severe headache occurred in 5 patients. Vomiting, nausea, and unsteady gait occurred in 2 patients. One patient had progressive limb weakness, and another had bulging eyes. All five patients underwent craniotomy. All five patients also underwent CT and MRI examinations. The diagnosis of CEICH was confirmed by pathology in 5 patients.

\section{CT scan}

CT was conducted with a 64-slice device and a 64-slice dual-source device (Siemens Company). The scanning parameters were as follows: $120 \mathrm{kV}, 420 \mathrm{mAs}$; scan time: 6.82s; thickness: $7 \mathrm{~mm}$; pitch: $0.8 \mathrm{~mm}$. In single-enhancement scanning, we used Omnipapue $(300 \mathrm{mg} / \mathrm{ml}, 80 \mathrm{ml})$ as a contrast agent, at an infusion rate of $2 \mathrm{ml} / \mathrm{s}$.

\section{MRI scan}

We used 1.5T and 3.0T scanners (GE TWIN SPEED superconducting magnetic resonance machine, Philips Company). Routine scans were performed with a standard head coil for axial, sagittal, and coronal scans. The following parameters were used: TIWI: TR $3000 \mathrm{~ms}$, TE 80 ms; T2WI: TR
$2000 \mathrm{~ms}$, TE $800 \mathrm{~ms}$; TlWI + C: TR $3000 \mathrm{~ms}$, TE $80 \mathrm{~ms}$; SWI: TR $16 \mathrm{~ms}$, TE $23 \mathrm{~ms}$, matrix 256 \pm 256 , slice thickness $5 \mathrm{~mm}$. Contrast-enhanced scans were acquired with Gd-DTPA as the contrast agent with the following parameters: TIWI: TR $500 \mathrm{~ms}$, TE $30 \mathrm{~ms}$; T2WI: TR $2000 \mathrm{~ms}$, TE 60, 80, 120 ms. Two patients underwent routine SWI with a standard head coil for axial scans with the following parameters: TR $16 \mathrm{~ms}$, TE $23 \mathrm{~ms}$, matrix $256 \times 256$, and slice thickness $5 \mathrm{~mm}$.

\section{Results}

\section{CT data}

CT showed quasi-circular or elliptical lesions with clear borders in five patients and hypodense lesions, representing edema that was surrounding the central lesion, with iso-density or low-density in 2 patients (Figure lA). CT in one patient showed stratification and mass effect. After administration of a contrast agent, CT showed mild ring enhancement in 2 patients, and moderate ring enhancement in 3 patients; the "ring" was smooth in contour (Figure 1B). The nature of the lesion was not diagnosed preoperatively.

\section{MRI data}

MRI of 5 cases showed an envelope with uniform thickness (measuring 1-3 mm). TIWI showed equal or slightly lower signal for the envelope, while the center of the lesion showed uniform high-signal intensity (Figure 2A). On T2WI, the envelope had low signal, and the center had uniform high signal (Figure 2B). The envelope was clearer with a thickness of about 1-3 mm. One case underwent SWI that showed a ring-like envelope more clearly, with low-signal intensity (Figure 3).

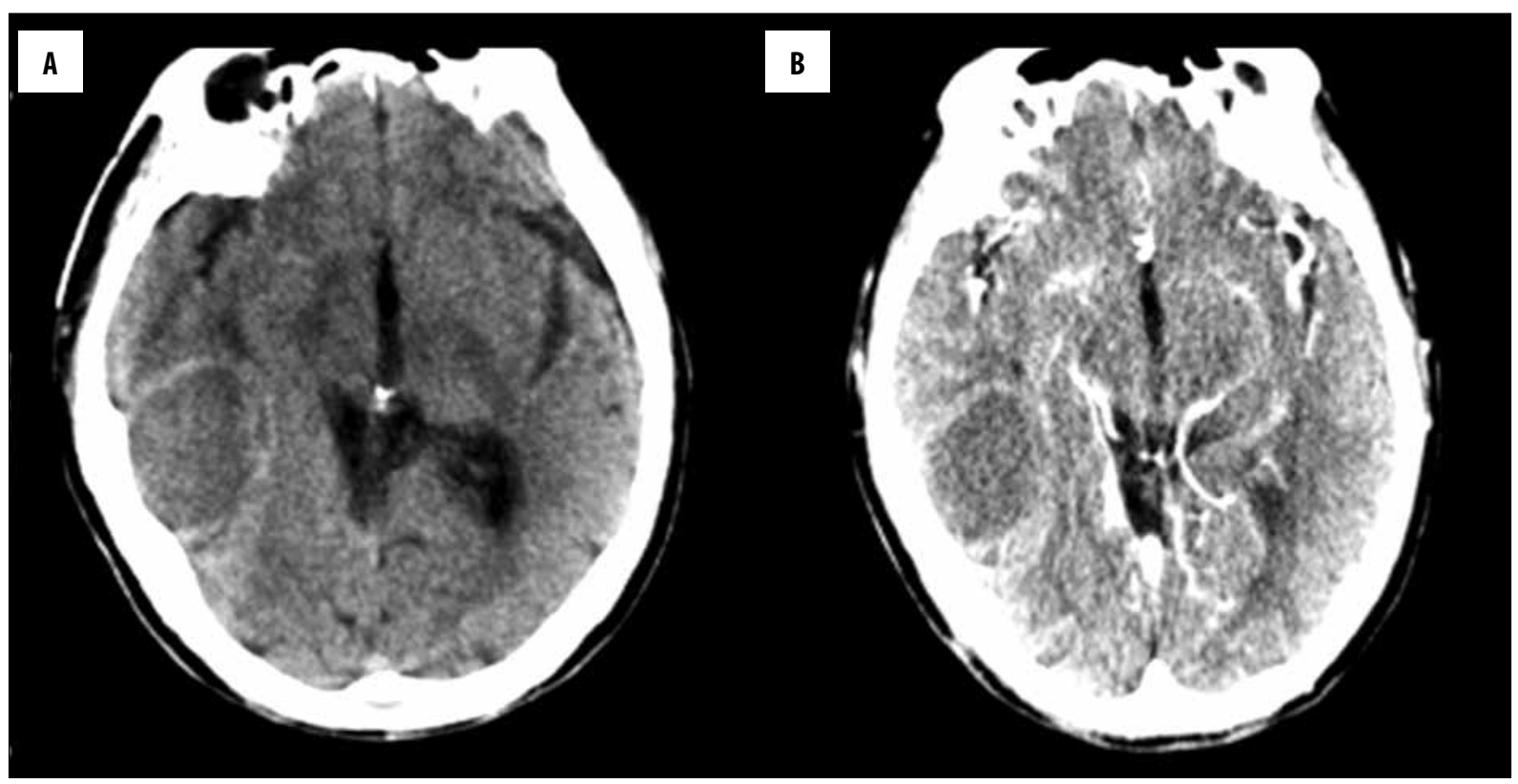

Figure 1. CT of CEICH. (A) CT scan shows quasi-circular, low density lesion with clear borders in the left temporal lobe of the brain that causes occlusion of the right occipital horn. (B) Enhanced CT shows "ring" enhancement. 


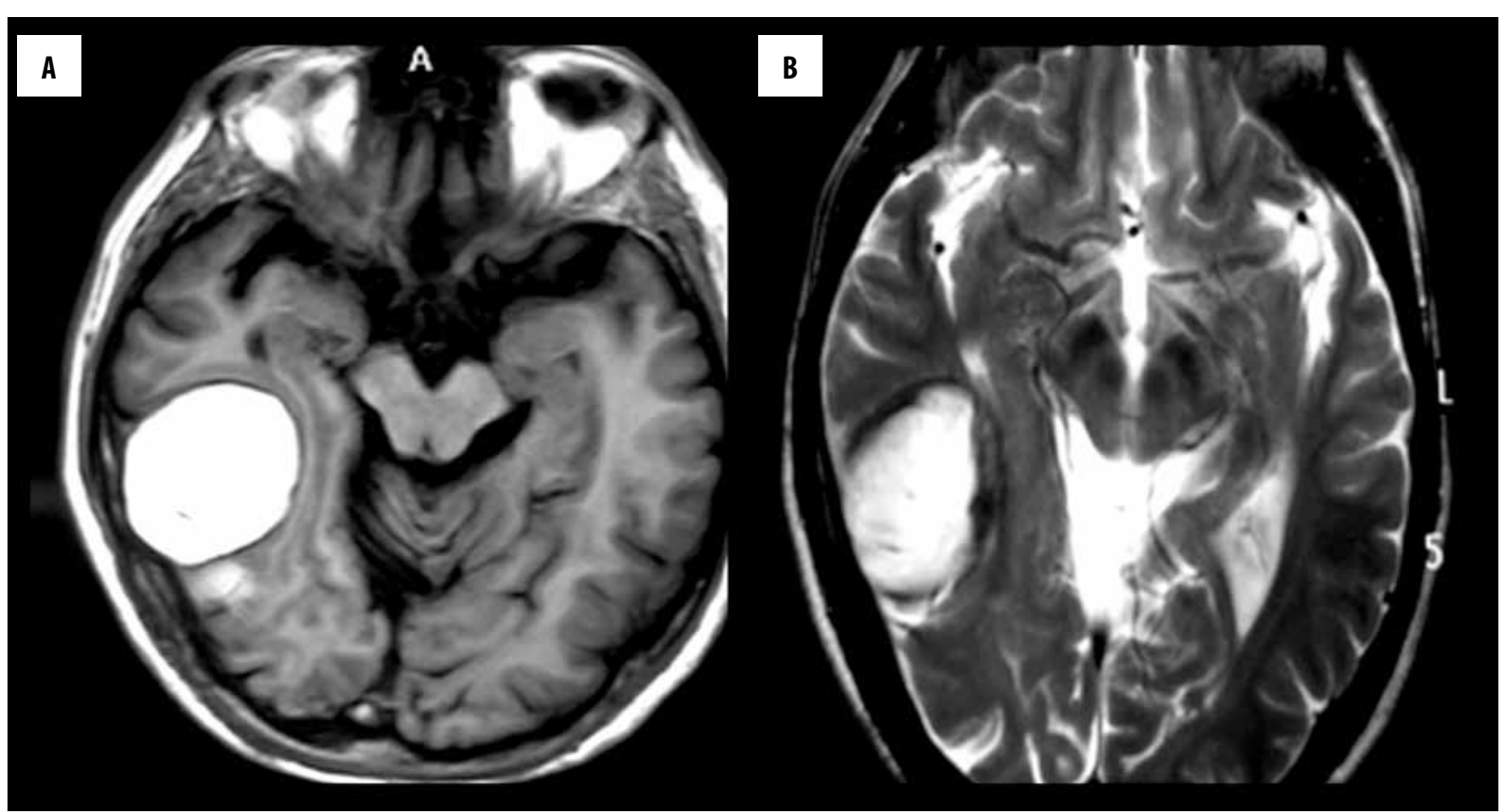

Figure 2. MRI of CEICH. (A) T1WI showed slightly lower signal for the envelope without edema. (B) T2WI shows a low-signal envelope and uniform high-signal center.

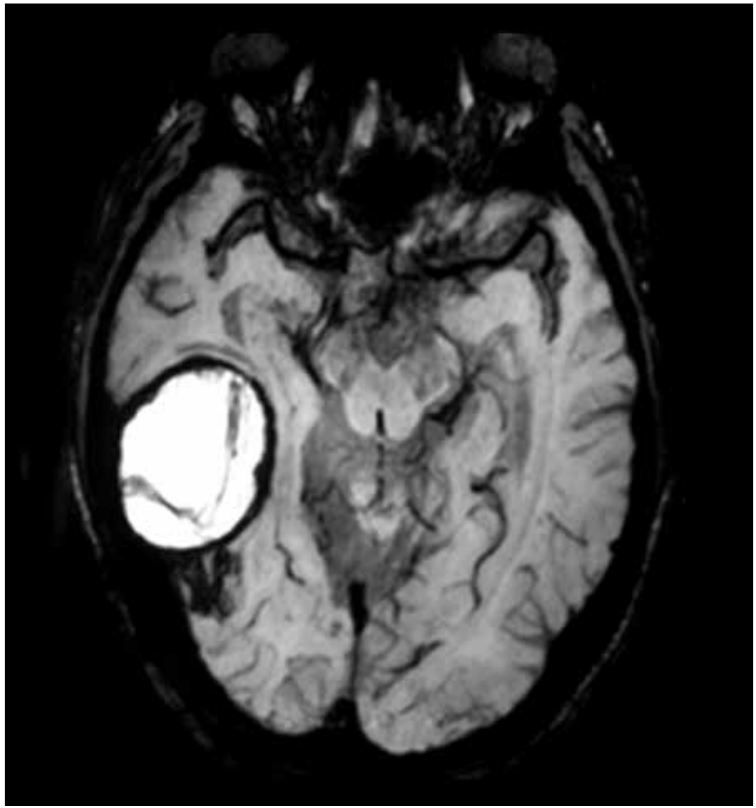

Figure 3. SWI of CEICH. A low-signal, ring-like envelope.

\section{Surgical and pathological findings}

Five patients underwent craniotomy. The hematomas were removed with complete capsules. The envelopes in 5 cases were thick and tough. The contents of hematomas had undergone complete degradation and liquefaction. Under the microscope, the capsules exhibited numerous blood vessels with varying thicknesses. Intravascular thrombus formation was noted in some of the blood vessels. Large amounts of fresh or shrunken red blood cells and large phagocytic cells containing hemosiderin were observed in both the liquefied hematomas and the blood clots. On the pathological examination, all cases were diagnosed as
CEICH. Two cases were diagnosed as hematomas with surrounding arteriovenous malformations.

\section{Discussion}

Intracranial hematomas are usually absorbed slowly or occasionally develop into cysts after a few months [3]. Trauma and vascular malformations, especially occult vascular malformations, are associated with the formation of CEICH. Small aneurysms, cerebral vascular hyalinization, cerebral arteriosclerosis, coagulation disorders, chronic inflammation, and even arteriovenous malformations (AVM) after radiotherapy can also develop into CEICH. In addition, there are numerous cases with an unknown etiology. In this study, 3 cases developed CEICH after craniotomy for arteriovenous malformation (AVM) and an aneurysm, which suggests postoperative trauma as a potential cause of CEICH in these cases.. Therefore, if cystic lesions appear in the vicinity of the surgical area after craniotomy, CEICH should be considered.

CT images of CEICH always show quasi-circular or elliptical cystic lesions. Most of the cysts are of uniform low density. Cysts can vary depending on different manifestations and different periods of bleeding, and can be of high or mixed density. On enhanced CT, granulation tissue with rich neovascularization can be observed as a ring enhancement pattern that is similar to that of brain abscesses $[4,5]$. Most lesions cause significant mass effect and cerebral edema around the lesion, which varies depending on the size and location of the lesion. However, the observations mentioned above are not specific characteristics of CEICH. Thus, CEICH is easily misdiagnosed as glioma, tumor, stroke, or brain abscess. A small proportion of capsular hematomas can be multi-lobed, and a few cases showed visible calcification $[5,6]$. Also, some studies reported that CEICH exhibited 2 components on initial CT, including an 
anterolateral fluid portion and a posteromedial solid portion [7]. In this study, lesions in the five cases appeared to be capsulated, with the intracapsular component showing uniform low density and high tension. It is therefore difficult to distinguish CEICH from brain abscesses.

Signal intensity of hemorrhage, that can be observed on MRI in patients with CEICH, varies chronologically, and it is complex. The capsule formation is mostly induced by subacute or chronic bleeding and therefore shows a high signal on TIWI and T2WI. The MRI signal of capsule can be high, isointense, or patchy isointense, when characterized by fresh bleeding. In the later stages of hematoma, long T1 and T2 signals can be observed on MRI, and the lesions look like cystic cavities containing cerebrospinal fluid. Macrophages in the hematoma can phagocytose, decompose, remove hemoglobin, and produce large amounts of hemosiderin. On TIWI and T2WI, cysts can be seen as surrounded by a ring-like envelope with low signal intensity. T2WIs show that more clearly as a black "ring" sign [4], which is specific for CEICH. In this study, old hemorrhage was found inside the envelope in 5 cases, where the hematomas were liquefied completely and mixed with plenty of fresh or shrunken red blood cells and large phagocytes containing hemosiderin. Three cases clearly showed the "ring" sign. SWI, in one case, clearly showed the lower signal "ring" sign. This may be due to the fact that the wall of the capsule contains hemosiderin, which affects the local magnetic field. No enhancement was found inside the lesions, but the envelope of the hematomas could have appeared to be annularly or linearly enhanced. Thus, observation of a hemosiderin ring and high intracapsular signals on MRI can point towards the diagnosis of CEICH.

Cerebral hemorrhage in CEICH is a slow and continuous process that is characterized by small amounts of bleeding in the initial stage [1]. Early symptoms are relatively mild and are often not noticed or misdiagnosed due to the lack of initial CT or MRI data. As the disease progresses and the capillaries bleed continuously, the hematoma continues to expand, and the mass effect increases gradually. Patients become concerned only when irritating symptoms are noticed or neurological damage occurs $[2,8,9]$. At this point, CT has no typical features. MRI features are also not conclusive but rather confusing, and cannot be recognized clearly, which often leads to misdiagnosis of glioma, metastasis, or brain abscess [2].

Since CT and MRI of CEICH are not performed on a regular basis in follow-up examinations, it is difficult to distinguish whether the lesion is the commonly seen intracerebral hematoma after absorption or a chronic intracerebral hematoma. However, large CEICHs must be resected. Thus, it is very important to distinguish these two types of hematomas. Intracerebral hematomas can be absorbed spontaneously and disappear completely after a few months [9].
CEICHs are characterized by gradually enlarged capsules and continuous bleeding [10]. Therefore, CT or MRI of CEICH shows high intracapsular signal and mass effect.

The clinical manifestations and CT and MRI characteristics of CEICH, brain tumors, stroke, and brain abscesses are highly similar. Therefore, misdiagnosis is very common. High signal intensity in the central area of the lesion on T1WI and T2WI, a low-intensity hemosiderin ring on T2WI or SWI, or coexistence of those signs could distinguish CEICH from brain tumors, stroke, and brain abscesses. The hematoma envelope ("ring") is intact in CEICH, but the ring in tumors and stroke is often partially or completely missing and is of an uneven thickness. This is because tumor invasion damages the blood-brain barrier, and phagocytes containing hemosiderin move away from the damaged blood-brain barrier. Brain abscesses usually exhibit uniform low signal intensity on TlWI, but some cases also can exhibit moderate or slightly higher signal intensity and lack of the "ring" sign. In conclusion, CEICH lacks specific characteristics. The "ring" wall and intracapsular bleeding features on MRI imaging reflect the pathological features of CEICH. Thus, MRI is an effective imaging modality for the diagnosis of CEICH.

It is still not entirely clear why CEICH hematomas are not absorbed. Most scholars $[4,11]$ believe that hematomas and their metabolites constantly stimulate the surrounding brain tissues, which causes inflammation in the surrounding brain tissue and gliosis. Gliosis and neovascularization, which form the hematoma capsule, constitute the capsule in CEICH as well. The surface of the capsule in CEICH often has abnormal vessels and capillaries that are continuously stimulated by inflammation. This leads to slow and persistent bleeding, and serum or plasma exudation, which allow hematoma widening and thickening of the wall of the capsule $[4,8,11]$. At the same time, activation of the VEGF pathway leads to neovascularization of the inner wall of the capsule, and blood cells leak into the hematoma cavity due to the large gap between cells in the walls of these newly formed vessels $[9,12,13]$. Therefore, the hematoma expands progressively. In this study, CT showed low density areas in the cysts, and no fresh bleeding was observed during surgery, suggesting that CEICHs bleed continuously in small amounts.

\section{Conclusion}

In conclusion, the "ring" and intracapsular bleeding characteristics that are found on MRI are helpful in the diagnosis of CEICH. In contrast, CT imaging of CEICH did not reveal any typical characteristics.

\section{Conflict of interest}

All authors declared no conflict of interest.

\section{References:}

\footnotetext{
1. Marutani A, Nagata K, Deguchi J et al: A Case of recurrent hemorrhages due to a chronic expanding encapsulated intracranial hematoma. Case Rep Neurol, 2015; 7: 173-80
}

2. Nishiyama A, Toi H, Takai H et al: Chronic encapsulated intracerebral hematoma: Three case reports and a literature review. Surg Neurol Int, 2014; 5: 88

3. Nomura M, Miyashita $\mathrm{K}$, Tamase A et al: A chronic intracerebral fluid hematoma. Neuroradiol J, 2014; 27: 191-94 
4. Ganapathy K: Chronic encapsulated intracerebral hematoma. Neurol India, 2011; 59: 504-5

5. Yuguang L, Liangwen Z, Fusheng L et al: Chronic expanding intracerebral hematoma. J Clin Neurosci, 2003; 10: 680-82

6. Lin CY, Chen Y, Tseng SH: Chronic encapsulated intracerebral haematoma. J Clin Neurosci, 2007; 14: 58-61

7. Fujii M, Takada Y, Ohno K et al: Slowly progressive expanding hematoma in the Basal Ganglia: A report of 3 cases and a literature review. Brain Nerve, 2012; 64: 295-302 [in Japanese]

8. Fiumara E, Gambacorta M, D'Angelo V et al: Chronic encapsulated intracerebral haematoma: pathogenetic and diagnostic considerations. J Neurol Neurosurg Psychiatry, 1989; 52: 1296-99

9. Nakamizo A, Suzuki SO, Saito N et al: Clinicopathological study on chronic encapsulated expanding hematoma associated with incompletely obliterated AVM after stereotactic radiosurgery. Acta Neurochir (Wien), 2011; 153: 883-93
10. Chen S, Zeng L, Hu Z: Progressing haemorrhagic stroke: Categories, causes, mechanisms and managements. J Neurol, 2014; 261: 2061-78

11. Ooba $\mathrm{H}$, Kamida $\mathrm{T}$, Isono $\mathrm{M}$ et al: Chronic intraventricular encapsulated hematoma presenting unique radiological features: A case report. Surg Neurol, 2003; 59: 23-27

12. Takeuchi S, Wada K, Sakakibara F, Mori K: Chronic encapsulated intracerebral hematoma associated with cavernous malformation. $\mathrm{J}$ Korean Neurosurg Soc, 2014; 55: 89-91

13. Takei J, Tanaka T, Yamamoto Y et al: Chronic encapsulated expanding thalamic hematoma associated with obstructive hydrocephalus following radiosurgery for a cerebral arteriovenous malformation: A case report and literature review. Case Rep Neurol Med, 2016; 2016: 5130820 